\title{
Témoignages écrits en langue vulgaire dans la Vallée d'Aoste du bas Moyen Âge
}

\section{Maria Costa}

\section{(2) OpenEdition}

1 Journals

\section{Édition électronique}

URL : http://journals.openedition.org/studifrancesi/9770

DOI : 10.4000/studifrancesi.9770

ISSN : 2421-5856

Éditeur

Rosenberg \& Sellier

\section{Édition imprimée}

Date de publication : 1 août 2017

Pagination : 289-294

ISSN : 0039-2944

\section{Référence électronique}

Maria Costa, "Témoignages écrits en langue vulgaire dans la Vallée d'Aoste du bas Moyen Âge », Studi Francesi [En ligne], 182 (LXI | II) | 2017, mis en ligne le 01 août 2018, consulté le 09 janvier 2021. URL : http://journals.openedition.org/studifrancesi/9770 ; DOI : https://doi.org/10.4000/studifrancesi.9770

\section{(c)}

Studi Francesi è distribuita con Licenza Creative Commons Attribuzione - Non commerciale - Non opere derivate 4.0 Internazionale. 


\section{Témoignages écrits en langue vulgaire dans la Vallée d'Aoste du bas Moyen Âge}

\section{Abstract}

Castles of the Aosta Valley preserve the most ancient proofs of the vernacular language practice. We are dealing with marked inscriptions on the walls of these dwellings: poetry or prose, locally originated or often "imported", that bear witness to the diffusion in the Aosta Valley of literary genres that belong to the French culture.

Also, the inventories of the castles reveal the presence of French literature works, such as the "mystères", the chivalric romances and the courtly literature.

The phenomenon of local variants, usually written by anonymous authors and inspired by works of French origin, is widespread: a fifteenth-century manuscript, conserved at Aosta Cathedral Archives, is a significant example.

Chronique de la Maison de Challant by Pierre du Bois is the first historical work written in French by a local author and it is dated from the second half of the fifteenth-century.

Dans le panorama culturel du bas Moyen Âge valdôtain, ce sont les châteaux qui jouent le rôle de protagonistes et non seulement du point de vue artistique, du moment qu'ils conservent les témoignages les plus anciens de la connaissance et de l'emploi de la langue vulgaire. Il s'agit premièrement de "graffiti" et inscriptions tracés avec les instruments les plus divers (couteaux, clous, craies d'argile ou terre rouge, morceaux de charbon, etc.), par des mains le plus souvent anonymes sur les murs de ces anciennes demeures seigneuriales. Ces empreintes, expression d'une attitude instinctive qui n'a pas cessé de séduire l'humanité depuis l'âge des cavernes, ont pu quelquefois résister aux injures du temps et aux intempéries, ainsi qu'aux multiples, successives transformations des manoirs - crépissages, restructurations, etc. - qui en ont, en plusieurs autres cas, effacé les traces.

C'est à partir de la fin du XIX ${ }^{\text {e }}$ siècle que les historiens locaux ont accordé de l'intérêt à cette typologie de sources, dont ils ont saisi l'importance historique. En fait, ces inscriptions témoignent d'une évolution linguistique qui, pour la Vallée d'Aoste, à travers son incorporation dans l'aire gallo-romaine avant et franco-burgonde après ${ }^{2}$, s'était développée pareillement à ce qui s'était passé dans les régions au-delà des Alpes Graies et Pennines, se renforçant à travers l'appartenance du diocèse d'Aoste à l'archidiocèse de Tarantaise, à partir du VIII ${ }^{\mathrm{e}}$ siècle, ainsi que, du point de vue politique et institutionnel, moyennant l'insertion dans ce puissant domaine que les comtes de Savoie avaient commencé à bâtir, depuis le XI ${ }^{\mathrm{e}}$ siècle, à califourchon des Alpes.

(1) Nous publions ici une synthèse des leçons consacrées à l'histoire de la langue et de la culture en Vallée d'Aoste, dans le cadre du Séminaire d'Histoire Valdôtaine institué par les Archives Historiques Régionales. Nous y avons fait amplement référence à l'ouvrage fondamental de L. ColliarD, La Culture valdôtaine au cours des siècles, Aoste 1976, pp. 1-75, auquel nous renvoyons le lecteur pour les approfondissements complémentaires.

(2) Fin du VI ${ }^{\mathrm{e}}$ siècle (575 après J.C.): $1^{\text {er }}$ royaume de Bourgogne; 1032: fin du dernier royaume de Bourgogne. 
Ce sont les chanoines François-Gabriel Frutaz (1859-1922) et Justin Boson (1881-1954) qui proposent à l'attention des érudits locaux, en les éditant, les graffiti découverts sur les murs des châteaux valdôtains: il s'agit de compositions poétiques ou en prose, tantôt de production locale, ou plus souvent d' "importation", qui témoignent en tout cas de la diffusion et de la circulation, en Vallée d'Aoste, de textes et genres littéraires appartenant à la culture française, que le passage de jongleurs et trouvères séjournant dans les châteaux avait contribué à faire connaître.

Parmi les compositions indiscutablement locales, il faut rappeler le célèbre quatrain, aujourd'hui malheureusement illisible, découvert à Fénis par l'abbé M. Durand et le chanoine F.-G. Frutaz, attribuant ces vers à Boniface de Challant, qui les aurait composés à l'occasion du mariage et du départ du château de sa fille Bonne, comme l'indique la date du 20 novembre 1402 clôturant ce bref poème chargé de nostalgie $e^{3}$.

A la littérature d'“importation" appartiennent, par contre, les "graffiti" du château de Quart, quatre vers en ancien français attribués par F.-G. Frutaz au roi de Navarre Thibaud de Champagne $(\dagger 1253)^{4}$; et encore les fragments du Roman de Renart, conservés au château de Châtillon, déjà signalés par J. Boson en $1921^{5}$, et les "Proverbes" de Fénis, édités par Boson même en 1919, qui ornent les fresques de la cour du château et constituent une variante locale de textes fort semblables que l'on retrouve dans plusieurs manuscrits français des XIV et XV siècles ${ }^{6}$.

Les "dits" et les "proverbes", espèces de sentences morales en vers inspirées de la vie quotidienne, au contenu savoureusement ironique sinon licencieux, constituaient en effet, à cette époque, un genre littéraire très cultivé et très populaire en France, à côté des "mystères", du roman chevaleresque et de la littérature courtoise. Ce qui reste ou dont on a pu reconstruire les traces, à travers les anciens inventaires des archives et des bibliothèques des châteaux valdôtains, atteste la présence d'ouvrages de la littérature française représentant presque tous les genres littéraires qu'on vient de citer: les fragments de l'Entrée d'Espagne, tirés d'une chanson de geste du $\mathrm{XIII}^{\mathrm{e}}$ siècle, retrouvés aux archives du château d'Entrèves à Châtillon et publiés par Paul Aebischer en 1925'; les deux fragments du Roman d'Escanor, appartenant au célèbre "cicle breton" ou "arthurien", retrouvés dans un manuscrit du XIII siècle provenant des archives Sarriod de La Tour et publiés par Joseph Bréan en $1948^{8}$; la composition titrée La chastelaine du Vergier, éditée à Florence en 1888 - luxueux tirage de 50 exemplaires - et republiée par Jules Brocherel en $1927^{9}$. Ce dernier ouvrage, tiré d'un manuscrit provenant de la bibliothèque du château d'Aymavilles et attribué à un anonyme écrivain valdôtain $\mathrm{du} X \mathrm{~V}^{\mathrm{e}}$ siècle, se révèle décidément intéressant $\mathrm{du}$ point de vue linguistique, car il constitue une variante locale en prose, riche d'expres-

(3) Edité in «Bulletin de l'Académie Saint-Anselme» (BASA), XXI, Aoste 1926, pp. 20-21; cf. aussi J. BRÉAN, Anthologie Littéraire Valdôtaine, Aoste 1948, p. 19; L. COLLIARD, La Culture cit., p. 25 et R. GoRRIS, Les débuts de la littérature valdôtaine francophone: du XV' au XVI siècle. «D'un château à l'autre...», in La littérature valdôtaine au fil de l'bistoire, Aoste 1993, p. 21.

(4) Cf. F.-G. Frutaz, Les origines de la langue française dans la Vallée d'Aoste, Aoste 1913, p. 43; J. BRÉAN, op. cit., p. 12; L. COLliarD, La Culture cit., pp. 19-20; R. GorRIS, art. cit., p. 21.

(5) J. Boson, L'apparition du français dans les actes publics du Duché d'Aoste, in «Augusta Praetoria» (AP), 5-6 (1921), p. 92.

(6) J. Boson, Proverbes en ancien français du château de Fénis, in AP, $4-5$ (1919), pp. 215-235; ID., Le château de Fénis, Novare 1953, pp. 15-30; L. Colliard, La Culture cit., pp. 22-25; B. Orlandoni-D. Prola, Il castello di Fénis, Aosta 1982, pp. $92-93$ et passim.

(7) P. AEBISCHER, Ce qui reste d'un manuscrit perdu de l'Entrée d'Espagne, in «Archivum Romanicum», t. XII (1928); L. COLliaRD, op.cit., p. 20.

(8) J. BrÉAn, Anthologie cit., pp. 223-227; L. Colliard, La Culture cit., p. 20.

(9) J. Brocherel, La chastelaine du Vergier, in AP, 1-12 (1927), pp. 1-17; L. Colliard, La Culture cit., pp. 39-44; R. GORRIS, art. cit., p. 23. 
sions dialectales, d'un ouvrage français, un roman courtois en vers composé dans la seconde moitié du XIII ${ }^{\mathrm{e}}$ siècle et dont l'action, centrée sur une aventure galante, se joue à la cour de Bourgogne.

Les anciens inventaires des châteaux recèlent, comme on l'a dit, des données intéressantes au sujet des goûts littéraires de l'aristocratie valdôtaine au bas Moyen Âge. Un document de ce type, parmi les plus riches et détaillés, est constitué par l'inventaire rédigé en 1565 à la mort du comte René de Challant. On y lit, par exemple, à propos des collections de livres conservées dans les châteaux de la famille Challant, que dans le "cabinet de la librairie" à Issogne on trouve deux volumes de Lancerot du lac, un livre de Petrarca réduit en françois et ung aultre intitulé Les Triomphes; à Aymavilles, la bibliothèque du "cabinet des droits" compte cinq livres, parmi lesquels ung livre escript à la main des afferes du roy Artus ${ }^{10}$.

Le phénomène des variantes locales, d'auteur le plus souvent anonyme, inspirées d'ouvrages d'origine française, est très répandu dans la littérature du Moyen Âge. La raison est double: tout d'abord il faut relever qu'il s'agissait de textes destinés à être représentés sur scène ou à être chantés par des trouvères itinérants qui s'accompagnaient d'un instrument musical; deuxièmement, il ne faut pas oublier qu'à cette époque on reproduisait n'importe quel ouvrage en le recopiant à la main. Tout cela ne pouvait que faciliter les improvisations et les adaptations, du point de vue linguistique ainsi que du contenu, qui pouvaient rapprocher davantage une œuvre littéraire du contexte local.

Un exemple très significatif, en ce sens, est constitué par un manuscrit du $\mathrm{Xv}^{\mathrm{e}}$ siècle, retrouvé aux Archives de la Cathédrale en 1986, lors du début des travaux de mise en ordre et inventoriage de ces archives. Il s'agit d'un petit cahier de 39 feuillets, contenant plusieurs textes soit en latin soit en français ${ }^{11}$. L'examen du cahier a révélé son appartenance au chanoine de la Cathédrale Louis de Saint-Pierre, qui en aurait rédigé la première partie, y ajoutant des textes composés par son oncle Jean de SaintPierre, lui aussi chanoine de la Cathédrale et curé de la paroisse de Morgex de 1434 à 1460; cette charge sera relevée justement par son neveu, Louis, jusqu'en 1499. Sans entrer dans le contexte d'une analyse minutieuse de ce manuscrit, qu'on a par ailleurs partiellement édité en $1987^{12}$, il faut en rappeler ici quelques compositions en vers qui constituent justement des variantes locales de textes déjà connus à travers des manuscrits français plus anciens. C'est le cas, par exemple, de la pièce qui porte le titre de Querela et qui est la version incomplète et légèrement modifiée d'une composition en vers connue en France sous le titre de La Complainte de Nostre-Dame tenant son chier filz entre ses bras, descendu de la croix; cette même conclusion s'applique à la composition titrée Les quinze signes du Jugement dernier, un poème connu en France déjà à partir du XII siècle et qui semble avoir joui d'une grande popularité au Moyen Âge: on en connaît en France 25 variantes, aucune desquelles ne correspond totalement au texte valdôtain conservé dans le manuscrit de la Cathédrale, qui représente donc, quoique lacuneux, la $26^{\text {ème }}$ variante. Ce détail est d'un intérêt extrême, notamment du

(10) Cf., à ce propos, M. CosTA, Indagine sui fondi librari dei secoli XV e XVI in Valle d'Aosta: elementi per lo studio delle collezioni private e delle biblioteche ecclesiastiche, in Conservazione dei materiali librari archivistici e grafici, textes réunis par Marina Regni e Piera Giovanna Tordella, vol. I, Torino 1996, pp. 341-345.

(11) Archives du Chapitre de la Cathédrale, Ms. Ex 78A. La reliure de ce cahier est constituée par un parchemin contenant un acte notarié rédigé un siècle avant par un notaire de Pré-Saint-Didier: le texte est malheureusement incomplet, puisque le parchemin a été recoupé et replié selon le format du manuscrit, un sort partagé, pendant tout le Moyen Âge et jusqu'à l'époque moderne, avec plusieurs autres parchemins de ce genre, "recyclés" pour servir de reliure lorsque leur primitive fonction n'avait plus d'intérêt.

(12) Cf. M. Costa, Un manuscrit latin-français du XV siècle des Archives capitulaires d'Aoste (EX $78 A$ ), in «Bibliothèque de l'A rchivum Augustanum» (BAA), Aoste 1987, pp. 5-41. 
point de vue linguistique, puisque les 25 copistes français ne semblent aucunement être originaires d'une région proche de la Vallée d'Aoste, la Savoie ou le Valais par exemple.

Deux autres pièces figurant dans le cahier de la Cathédrale posent davantage des problèmes: la première, Dit de don denier, reprend, mais seulement dans les deux premiers vers, un "proverbe" français au titre à peu près identique, célébrant sur un ton de satire l'apologie de l'argent, un sujet très cher à la littérature classique et du Moyen Âge. Le texte connu en France ${ }^{13}$, beaucoup plus long et modulé sur un ton de cantilène, est rythmé par la répétition des mots Denier est ou Denier fait au début de la plupart des vers. Plus synthétique mais plus mordant, le texte du manuscrit valdôtain, fait recours à un langage direct, quoique enrichi de similitudes et métaphores, pour attester que l'argent - "don denier" justement - est le protagoniste gagnant dans tous les domaines de la vie sociale: l'argent assure gain de cause dans n'importe quel procès et permet même de soustraire au gibet un homme déjà condamné; "don denier" fait célébrer des obsèques solennelles pour un riche bourgeois défunt; il dirige l'élection des papes et des cardinaux et peut transformer un berger en ecclésiastique et un paysan en chevalier; "don denier" permet aux femmes d'être très belles et élégantes. Pourtant, l'ironique conclusion de notre écrivain est celle-ci:

\section{Totes choses pout faire don denier, \\ For que grant cors et petit chier \\ Et bome mort resucité.}

Si l'argent peut tout faire, il n'a tout de même pas le pouvoir de faire devenir un corps plus grand ou une tête plus petite, ni de ressusciter un homme de la mort!

Une autre composition insérée dans le manuscrit de la Cathédrale constitue, à l'état actuel des recherches, une pièce unique, puisqu'on n'a trouvé son équivalent dans aucun manuscrit français jusqu'ici répertorié. Il s'agit du Débat entre Satan, les bommes et la Vierge, un texte vraisemblablement destiné à la représentation sur scène: on y rencontre plusieurs personnages - le Père éternel, la Vierge Marie, l'archange Gabriel, les hommes, le démon et ses acolytes - et on y représente symboliquement l'éternelle lutte de l'humanité contre les puissances du mal. L'action se déroule autour d'un tribunal imaginaire où le juge suprême, Jésus Christ, par l'intermédiaire de l'archange Gabriel, convoque l'humanité entière: le "Ministère public", représenté par Sathanas, en qualité de procureur de tous les démons de l'enfer, prétend tout court avoir gain de cause, mais les hommes choisissent pour leur défenseur la Vierge Marie, qui est la mère du Christ, porteuse donc de Celui qui rachète l'humanité de la condamnation éternelle. Quant à Sathanas, qui perd le procès, il n'a pas d'autre choix que de redescendre aux enfers, où il est pris pour cible des violentes protestations de ses semblables.

Le "débat" peut se rapprocher, comme genre littéraire, des fameux "mystères": nés comme compléments des rites célébrant les grandes fêtes liturgiques, ils se détachent progressivement du décor théâtral constitué par les églises ou leurs parvis, pour s'orienter de plus en plus vers des lieux profanes, les places, les rues, les grands carrefours. L'auteur de notre débat, composé de plus de 250 vers, demeure inconnu, bien qu'on ne puisse pas totalement exclure que le chanoine Jean de Saint-Pierre, qui

(13) Edité par A. JubINAL, Jongleurs et trouvères... des XIIt et XIV siècles, Paris 1835, réimpr. Genève 1977, pp. 94-100. Cf. aussi J.V. Molle, «De dan denier»: Contributo a un'edizione critica, in «Studi filologici e letterari», Genova s.d., pp. 221-255. 
clôture la composition avec les mots Istud romancium est nobili viro Johanni de Sancto Petro..., n'ait pas uniquement joué le rôle d'un simple copiste. Il est tout de même significatif que le manuscrit des Archives capitulaires d'Aoste renferme l'unique rédaction de ce texte connue jusqu'à présent.

Par contre, pour une autre pièce de théâtre, un "mystère" pour la précision, composée par un auteur anonyme de la seconde moitié du XV siècle, on peut parler d'un véritable "best-seller": il s'agit du Mystère de saint Bernard de Menthon, plusieurs fois représenté à Aoste et au Grand-Saint-Bernard, toujours avec un grand enthousiasme du public. La pièce reprend le canevas d'une biographie rédigée au XV siècle par un écrivain vraisemblablement savoyard et faussement attribuée à Richard de Valdisère, successeur de Bernard, à la mort du saint (1081), dans la charge d'archidiacre d'Aoste ${ }^{14}$. Le succès populaire de cette pièce relève du fait qu'elle reprend les éléments les plus légendaires et fantastiques relatifs à la vie de Bernard d'Aoste, à partir du célèbre récit de la lutte engagée par le saint au sommet du Mont-Joux contre les esprits démoniaques infestant le col, épisode qui a, entre autres, fortement inspiré l'iconographie populaire du saint.

Un autre genre littéraire strictement lié aux célébrations liturgiques est le "noël”, composition en vers accompagnée d'une mélodie, destinée à être chantée justement à l'occasion de la Noël. Entre le XV et le XVI siècle, les "noëls" connurent en France une grande popularité, au point qu'on en a publié, il y a quelques dizaines d'années, plusieurs recueils et de nombreuses études critiques leur ont été consacrées. Les deux derniers feuillets du manuscrit de la Cathédrale qu'on vient d'examiner contiennent quelques strophes d'un célèbre "noël" dont on connaît au moins deux autres versions plus anciennes, accompagnées aussi de la notation musicale, conservées dans les mss. 11 et 13 de la Bibliothèque du Grand Séminaire d'Aoste. Cette composition présente un texte bilingue: une partie en latin, confiée probablement à la voix du célébrant ou d'un chantre, et une partie en langue vulgaire, en l'espèce un mélange de langue d'oïl et de formes franco-provençales, destinée au chœur ou à l'assemblée des fidèles. Pour l'analyse philologique et des contenus de ce "noël", on renvoie à l'étude critique de Gianni Mombello, qui suggère pour cette composition une origine valdôtaine ${ }^{15}$.

Après ce riche panorama de textes littéraires, l'histoire ne pouvait pas faire défaut. Dans la seconde moitié du XV $\mathrm{X}^{\mathrm{e}}$ siècle on trouve le premier ouvrage de chronique historique, en langue française, d'auteur valdôtain: l'écrivain en question est Pierre du Bois, appartenant à une riche famille de marchands originaire d'Aymavilles. Il est marchand lui-même et, en même temps, secrétaire personnel du comte Jacques de Challant, seigneur d'Aymavilles, charge qu'il remplira jusqu'à la mort de ce dernier, le 14 juin 1459.

Peu de temps après, Pierre du Bois termine un ouvrage titré Chronique de la Maison de Challant, dans lequel il passe en revue presque deux siècles et demi d'histoire de cette famille noble, avec une attention prioritaire à la branche d'Aymavilles et surtout à Jacques de Challant, dont l'auteur veut, évidemment, célébrer les vertus et la renommée. L'ouvrage de Pierre du Bois est en prose, mais il se termine par deux ballades en vers que l'Auteur dédie aux armoiries de son seigneur et à la grandeur de la

(14) Le mystère a été édité par A. LECOY DE LA MARCHE, Le Mystère de saint Bernard de Menthon, Société des anciens textes, Paris 1889, éd. anastatique Aoste 1983, avec préface de L. Colliard. Pour un examen critique de cette pièce cf. P. AEBISCHER, Le «Mystère de Saint Bernard de Menthon» in AP, 4-5-6 (1925), pp. 49-61; cf. encore L. COLLIARD, La Culture cit., pp. 34-39.

(15) Cf. G. Mombello, Analyse philologique d'un noël conservé dans deux manuscrits du Grand Séminaire d'Aoste, in Le culte et ses rites: des témoins manuscrits aux expressions de la dévotion populaire, Actes du Colloque international d'Aoste (2 et 3 avril 1993), réunis par M. Costa, Aoste 1994, pp. 169-213. 
Maison de Challant. Cette deuxième ballade se compose de quatre strophes - quatre huitains et un quatrain - se terminant chacune par l'acclamation Vive Challant, une acclamation répétée, de nos jours encore, lors du Carnaval historique de Verrès, bien qu'elle se rapporte aujourd'hui aux adversaires de Jacques, Catherine de Challant et Pierre d'Introd ${ }^{16}$.

Ce n'est certainement pas l'unique lien que les modernes célébrations du Carnaval ont gardé avec des personnages et des thèmes de la littérature du bas Moyen Âge: que l'on songe à l'omniprésence du diable et aux représentations du Jugement dernier que l'on jouait encore, il y a quelques décennies, dans la Vallée d'Ayas.

On a cité, jusque-là, uniquement des exemples littéraires qui témoignent de l'emploi du français en Vallée d'Aoste, mais il y a d'autres traces écrites, quoique plus rares, qui attestent l'usage de la langue vulgaire en dehors des châteaux, des cours féodales et des milieux ecclésiastiques. L'exemple le plus ancien, en ce sens, se situe dans le domaine de la vie administrative: il s'agit d'un sceau pendant attaché à un parchemin qui contient un document de l'année 1374; le sceau porte les armoiries des seigneurs de Quart et présente sur le contre-sceau une inscription en langue vulgaire, Henri sire de Quart, qui fait pendant avec une inscription analogue en latin placée le long du périmètre de ce même sceau. Le cas du seigneur Henri de Quart qui choisit d'adopter le français dans l'inscription de son contre-sceau, précède de presque deux siècles le fameux édit d'Emmanuel-Philibert (22 septembre 1561) qui prescrit l'emploi de la langue française dans les différents secteurs de la vie publique et il constitue, pour le XIV ${ }^{\mathrm{e}}$ siècle, à l'état actuel des recherches, un cas unique ${ }^{17}$.

En conclusion de cet excursus, nous sommes tout de même obligés de revenir encore aux châteaux, en restant toutefois dans le contexte de la vie quotidienne.

Le château d'Issogne a gardé, au cours des siècles, plus que tout autre manoir valdôtain, un corpus d'inscriptions spontanées et graffiti tracés par la main des habitants de cette demeure, nobles et domestiques, ainsi que par les hôtes du château: représentants de la noblesse et du clergé, notables, mais aussi ouvriers, artistes et artisans, voyageurs et pèlerins, provenant des différents pays de l'Europe occidentale. Si l'emploi du latin est encore très fréquent dans les inscriptions qui remontent aux $\mathrm{XV}^{\mathrm{e}}$ et $\mathrm{XVI}^{\mathrm{e}}$ siècles, et notamment en ce qui concerne les emprunts aux œuvres classiques ou aux textes sacrés, les langues vulgaires font également leur apparition dans les graffiti se rapportant à la vie quotidienne ${ }^{18}$. Nous ne rappellerons ici, parmi ces derniers, que le plus ancien, datant du $\mathrm{XV}^{\mathrm{e}}$ siècle: 1489. Jan de Valupe a faict la cave de ce chateaus pour 20 florin(s). L'importance de cette inscription, non seulement du point de vue linguistique, mais en ce qui concerne aussi l'histoire de la construction du château est évidente. Les exemples datant du Xvi siècle et témoignant de l'usage de la langue vulgaire sont beaucoup plus nombreux, mais leur examen dépasserait les limites chronologiques imposées par ce bref aperçu. Nous renvoyons toutefois le lecteur à la belle étude critique que O. Borettaz a consacrée aux graffiti du château d'Issogne, ouvrage qui présente, entre autres, le catalogage de presque 600 inscriptions.

MARIA COSTA

(16) L'œuvre de Pierre du Bois a été éditée par O. Zanolli, in «Archivum Augustanum» (AA), IV, Aoste 1970, pp. 1-136. Cf. aussi L. Colliard, La Culture cit., pp. 27-34.

(17) Cf. à ce propos, J.-G. Rivolin, Un important document linguistique: le sceau d'Henri de Quart (1374), in «Le Flambeau» 3 (1989), pp. 5-7.

(18) O. BoretTAz, I graffiti nel castello di Issogne in Valle d'Aosta, in «Quaderni di cultura alpina» 46, Aosta 1995. 